Annuaire suisse de politique de développement

2 | 1982

Annuaire Suisse - Tiers Monde 1982

\title{
La Coopération de la Suisse au développement : politique et réalisations
}

Die Schweizerische Entwicklungszusammenarbeit: Verwirklichungen

Jacques Forster

\section{CpenEdition}

Édition électronique

URL : http://journals.openedition.org/aspd/1132

DOI : $10.4000 /$ aspd. 1132

ISSN : 1663-9669

Éditeur

Institut de hautes études internationales et du développement

\section{Édition imprimée}

Date de publication : 31 août 1982

Pagination : 181-206

ISSN : 1660-5934

\section{Référence électronique}

Jacques Forster, «La Coopération de la Suisse au développement : politique et réalisations », Annuaire suisse de politique de développement [En ligne], 2 | 1982, mis en ligne le 23 janvier 2013, consulté le 08 septembre 2020. URL : http://journals.openedition.org/aspd/1132 ; DOI : https://doi.org/10.4000/ aspd. 1132 


\title{
LA COOPERATION DE LA SUISSE AU DEVELOPPEMENT : POLITIQUE ET REALISATIONS *
}

\author{
Jacques Forster
}

\section{DIE SCHWEIZERISCHE ENTWICKLUNGSZUSAMMENARBEIT : VERWIRKLICHUNGEN}

Zusammenfassung : Dieser Bericht vermittelt einen umfassenden und doch kurzen Ueberblick über die schweizerische Politik der Entwicklungszusammenarbeit und deren seit ihrem, im Vergleich mit anderen Industrieländern, relativ späten Entstehen. Die der Entwicklungspolitik - der humanitäre Grundgedanke und die Vertidigung schweizerischer Interessen - und deren mitunter konfliktträchtigen Synthese zu einem Instrument der Förderung eigenständiger Entwicklung der Entwicklungsländer sind dargestellt und, besonders in ihrer Evolution der 70er Jahre, auseinandergesetzt. Der politischen Analyse sind dann die Ausführungsprinzipien, Formen, Schwergewichte, Verteilung und quantitative Evolution der tatsächlichen Entwicklungsleistungen der Schweiz gegenübergestellt. Die Schlussfolgerung blickt in die Zukunft : infolge ihrer Verflechtungen hat die Schweiz ein grundsätzliches und wichtiges Interesse an der Fortführung einer verbesserten Entwicklungshilfe und -zusammenarbeit. Die Möglichkeiten dafür bestehen heute, schon weil die Oeffentlichkeit besser und umfassender über die Wichtigkeit der Nord-Südbeziehungen informiert ist. Es bestehen heute zwar wenig Möglichkeiten für eine erhebliche Verbesserung der quantitativen Leistungen, doch besteht noch Spielraum in quantitativer Hinsicht (Ungebundenheit der Hilfe, bessere finanzielle Bedingungen, vermehrte Beteiligung an den lokalen und laufenden Kosten von Entwicklungsprojekten). Trotz des gespannten und reichlich debattierten Beziehungsfeldes der Aussenwirtschafts- und der Entwicklungspolitik kann man heute einen Ausbau wirtschaftlicher Instrumente des Entwicklungszusammenarbeit feststellen, und eine Beibehaltung des entwicklungspolitischen Gleichgewichtes der vergangenen Jahre scheint möglich zu sein.

L'aide publique au développement (APD) ne représente qu'une faible partie des relations économiques entre les pays du Nord et ceux du Sud. Vu I'opa-

* Cet article est basé sur une communication présentée en juin 1982 à Oslo lors d'un colloque de I'EADI sur les politiques et réalisations des pays européens en matière de coopération au développement. 
cité qui caractérise trop souvent les autres flux financiers, on serait même tenté de dire qu'elle ne représente que la partie visible de l'iceberg. Cette image sied bien au cas de la Suisse, pays où les flux de capitaux privés vers les pays du Tiers Monde sont particulièrement importants, mais où, par contre, I'APD est particulièrement modeste. Quels sont les éléments de cette situation d'autant plus remarquable que la Suisse est en tête du palmarès du PNB par habitant?

Un premier trait est la forte intégration de la Suisse à l'économie mondiale : les exportations de biens et de services non facteurs représentaient en 1979 pas moins de $35 \%$ du PIB, alors que la moyenne pondérée correspondante pour l'ensemble des pays industriels à économie de marché était de $19 \%$ (1). La Suisse est une des principales places financières du monde; c'est une plaque tournante importante, notamment pour les opérations sur les eurodevises, et ses entreprises investissent beaucoup à l'étranger, mettant la Suisse au troisième ou quatrième rang mondial pour ce qui est de l'investissement direct.

Les relations avec les pays du Tiers Monde revêtent de plus en plus d'importance pour l'économie suisse; la part de ses exportations vers le Tiers Monde représente un cinquième environ de ses exportations totales, le solde de la balance commerciale avec ces pays étant largement positif. On estime qu'environ deux cent mille emplois en Suisse sont liés aux exportations vers les pays du Tiers Monde. Par rapport à la dimension de son économie, la Suisse est le plus gros exportateur de capitaux vers ces pays $(3,1$ milliards de dollars en moyenne par année de 1976 à 1980, soit 3,81\% du PNB; la moyenne des pays membres du CAD étant de 0,68\% du PNB) (2). Les principaux échanges économiques ont lieu avec un relativement petit nombre de pays, qui sont surtout des exportateurs de pétrole ou de nouveaux exportateurs d'articles manufacturés. Les investissements directs se concentrent essentiellement en Amérique latine (Brésil, Argentine, Mexique).

Sur le plan historique, il faut rappeler que la Suisse, comme d'autres petits pays européens, n'a pas eu de liens coloniaux de type politique avec les pays du Tiers Monde. Ce fait a eu deux conséquences sur l'aide publique au développement :

- un démarrage assez tardif et lent puisqu'il fallut, lors de la grande vague de décolonisation politique autour des années 1960-61, créer des organismes chargés de gérer les programmes de coopération technique et financière;

- l'absence du poids des relations coloniales dans la répartition géographique de l'aide.

Sur le plan des institutions politiques, la Suisse présente deux caractéristiques originales qui doivent être mentionnées dans le présent contexte :

- tout d'abord, il semblerait que la Suisse soit le seul pays membre du CAD dans lequel une votation populaire ait eu lieu dans le domaine qui nous 
intéresse. L'issue négative du scrutin de 1976 sur l'octroi du prêt de deux cents millions de francs à I'IDA aura - malgré I'ambiguïté des conclusions que I'on a pu en tirer - pesé lourdement sur la politique gouverne mentale de coopération au développement;

- en deuxième lieu, mentionnons que le gouvernement de la Confédération est une coalition des quatre principaux partis politiques. Ces quatre partis représentent plus de $80 \%$ des sièges dans les deux chambres du Parlement. Jusqu'ici, la politique définie par le Conseil fédéral a bénéficié, dans toutes les décisions importantes, du soutien de tous les partis gouvernementaux. Ces derniers n'ont pourtant pas la même conception de cette dimension de la politique extérieure (3). C'est dire que dans sa formulation et son application, cette politique reçoit I'approbation d'un éventail politique beaucoup plus ouvert que dans d'autres pays où les coalitions gouvernementales sont en général moins larges et plus homogènes. C'est un trait dont il faut tenir compte dans l'analyse de la politique suisse de coopération au développement.

\section{Les objectifs généraux de la coopération au développement : le discours officiel}

Depuis 1971, le Conseil fédéral a eu maintes occasions de présenter et de défendre sa politique de coopération au développement et d'en préciser les objectifs :

- à plusieurs reprises, il a adressé à l'Assemblée fédérale des demandes de crédits pour la poursuite de la coopération technique et financière, pour le financement de mesures de politique économique et commerciale, pour la participation à diverses actions de caractère multilatéral;

- en 1973 et en 1975, il proposait au Parlement deux rapports à I'appui d'un projet de loi destiné à donner une base juridique à cette coopération, qui devenait une nouvelle dimension permanente de la politique extérieure helvétique;

- enfin, en 1976, la votation populaire sur un crédit de deux cents millions de francs à I'IDA fut l'occasion d'un. vaste débat dans lequel le gouvernement eut à intervenir pour réaffirmer sa politique et la défendre face aux critiques émanant aussi bien de la droite que de la gauche.

Une doctrine se dégage de ces différents textes gouvernementaux faisant apparaître les deux objectifs généraux de la coopération au développement - la promotion du développement des pays du Tiers Monde et la défense des intérêts économiques et politiques extérieurs de la Suisse - comme les deux faces d'une même médaille.

\section{La défense des intérêts de la Suisse}

Dans le discours gouvernemental, deux motivations essentielles apparaissent chaque fois qu'il s'agit de justifier la coopération au développement : le 
devoir moral d'un pays riche confronté à la misère de centaines de millions d'hommes, et l'intérêt politique et économique national.

La motivation humanitaire est l'issue d'une tradition née avec Henri Dunant, fondateur de la Croix-Rouge en 1864, et qui se poursuit au XXe siècle par des interventions du gouvernement dans le domaine diplomatique et financier. La motivation d'ordre humanitaire est bien comprise et admise par I'opinion publique car elle correspond bien à l'image que la Suisse se plaît à donner à l'extérieur : un pays certes privilégié, mais généreux et ouvert aux problèmes mondiaux. Cette motivation est d'ailleurs partagée par l'ensemble des forces politiques du pays, y compris l'extrême droite qui, bien qu'hostile à la coopération au développement, est favorable à l'aide de caractère humanitaire (4).

Les motivations liées à la défense des intérêts nationaux - économiques et politiques - occupent une place importante dans le discours. On considère que la prospérité de la Suisse est liée à l'intensité de ses relations économiques extérieures; ces dernières sont tributaires de la stabilité d'un système économique mondial fondé sur la libre circulation des biens, des services et des capitaux. La défense des intérêts nationaux passe donc par la défense de ce système :

"Pour que la Suisse puisse retirer pleinement avantage, en vue du bien-être de sa population, des relations économiques, il faut qu'elle puisse continuer à participer à un système économique mondial aussi libéral que possible, auquel les pays en développement soient intégrés et dont ils puissent eux aussi profiter. Ils ne pourront toutefois y parvenir qu'à la condition de faire progresser leur développement économique et social. C'est la raison pour laquelle nombre d'entre eux, dans la difficile situation qu'ils connaissent actuellement, ont besoin d'un soutien accru" (5).

A des niveaux plus particuliers, I'analyse du discours gouvernemental met en lumière la place de plus en plus importante occupée par les pays du Tiers Monde dans l'économie mondiale, en tant qu'exportateurs non seulement de produits de base, mais aussi de produits manufacturés, et en tant que clients des industries d'exportation des pays industrialisés.

Le rôle de la coopération au développement est donc d'éviter que des crises économiques $n$ 'entraînent des pays du Tiers Monde "à réduire leurs échanges commerciaux avec nous, à ne plus pouvoir rembourser leurs dettes, à renoncer à nos investissements et à notre technologie, enfin à se couper des pays industrialisés" (6). "Il appartient à la coopération suisse au développement de maintenir et de renforcer la capacité d'absorption des pays en développement pour ce qui est des produits d'exportation et des investissements suisses" (7).

Mais les relations avec les pays du Tiers Monde ne sont pas seules en cause. La coopération au développement doit tenir compte du contexte des relations de la Suisse avec les autres pays industrialisés. "Par notre contribution, nous concourrons à répartir de façon adéquate entre les pays industrialisés 
le poids du soutien qu'il est nécessaire de donner aux pays en développement. Notre pays ne saurait, sans se causer du tort, refuser de faire sa part d'une tâche commune" (8). Le même raisonnement vaut dans les relations de la Suisse avec les organisations internationales : c'est en collaboration avec elles que la Suisse pourra défendre ses propres intérêts et les protéger au sein de ces organisations. Dès 1976, cette argumentation repose sur un exemple concret : le refus de la Suisse - après une votation populaire - de contribuer à la reconstitution des ressources de I'IDA placera constamment le gouvernement dans une attitude défensive vis-à-vis du groupe de la Banque mondiale. En 1980, le président de la BIRD, Mc Namara, lors d'une visite en Suisse, ne manquait pas de relever la dépendance de la Suisse à l'égard du Tiers Monde et insistait sur la nécessité pour la Suisse d'aider davantage les pays en développement et... le groupe de la Banque mondiale (9).

II faut finalement relever que si le terme de "coopération au développement" est constamment utilisé dans le discours gouvernemental pour bien illustrer la communauté d'intérêt entre pays du Tiers Monde et pays industrialisés, l'attention du Conseil fédéral est essentiellement concentrée sur le volume de I'APD. Trois raisons à cela :

a) I'APD est - dans la situation actuelle - le principal indicateur quantifia-

ble de la sensibilité d'un gouvernement aux problèmes du développement; or la Suisse est mal placée à cet égard. C'est sur ce point que les pays industrialisés sont le plus critiques à l'égard de la Suisse et qu'ils exercent une pression diplomatique afin qu'elle augmente rapidement le volume de son APD;

b) faute de cette aide, un grand nombre de pays ne seraient pas en mesure d'améliorer leur situation,

c) “contrairement à d'autres mesures que réclament aujourd'hui ces pays, les transferts de ressources ne remettent pas en cause les structures économiques internationales" (10).

Dans l'analyse qui précède, il est question de l'intérêt général et à long terme de l'économie suisse à pouvoir bénéficier de la stabilité du système de coopération économique mondial et du développement général de pays qui deviendraient ainsi de meilleurs partenaires économiques. Qu'en est-il des intérêts à court terme? L'aide est-elle directement un instrument de promotion des exportations? Voici la réponse du Conseil fédéral :

"Certaines de nos activités en matière de coopération technique et d'aide financière profitent directement à notre économie. C'est le cas lorsque du matériel et de l'équipement suisses sont achetés dans le cadre de nos projets de coopération technique ou d'aide financière. Plus généralement, notre économie bénéficie aussi de l'aide financière accordée aux pays en développement par l'ensemble des pays industrialisés et des pays de I'OPEP ainsi que par les organisations internationales. En augmentant les ressources financières des pays en développement, en atténuant la pression exercée sur leur balance de paiements, I'aide publique permet à ces pays d'investir davantage et de continuer à acheter dans les pays développés, ce dont notre indus- 
trie profite. Mais notre volonté d'aider les pays en développement n'est pas dictée au premier chef par la perspective de retirer des avantages immédiats; ils n'ont notamment pas d'influence sur la façon dont nous accordons notre aide, ni sur la manière dont nous préparons nos projets de coopération technique et d'aide financière dont les seuls objectifs restent ceux qui sont fixés dans la loi (cf. infra). Néanmoins, il s'agit d'avantages qui, dans une certaine mesure, sont une compensation directe de l'effort que nous consentons à faire" (11).

Pour le Conseil fédéral, la coopération au développement contribue aússi au maintien de la paix: "elle veut contribuer à atténuer certaines des inégalités qui existent entre les membres de la communauté internationale ainsi que les tensions et les dangers de conflit qui en résultent" (12). La coopération au développement est ainsi une contribution à la sécurité de la Suisse; cet argument fut évoqué à nouveau en 1980 lors d'un débat parlementaire.

Et le Conseil fédéral conclut : "Que l'on aborde le problème sous l'angle de notre devoir moral de pays riche, sous celui des impératifs de notre politique étrangère ou encore sous le rapport des objectifs de notre politique économique extérieure, la même conclusion s'impose : nous devons accroître notre aide publique au développement et nous y avons intérêt" (13).

Dans les textes qui précèdent, où chaque mot est pesé, on peut lire un discours s'adressant à deux publics bien différents : ceux qui voudraient une coopération au développement dégagée des contingences de la défense des intérêts nationaux et ceux qui ne voient dans cette activité relativement nouvelle du gouvernement que la poursuite de la politique économique extérieure par d'autres moyens.

\section{La promotion au développement}

La formulation la plus condensée - et la plus autorisée - des objectifs de la coopération suisse au développement, par rapport à la situation des pays du Tiers Monde, est sans aucun doute celle de la loi sur la coopération au développement et l'aide humanitaire internationale du 19 mars 1976. Son article 5 énonce ainsi les buts de la coopération au développement :

a) "La coopération au développement soutient les efforts des pays en développement en vue d'améliorer les conditions de vie de leurs populations. Elle doit contribuer à mettre ces pays en mesure d'assurer leur développement par leurs propres forces. Elle tend, à long terme, vers un meilleur équilibre au sein de la communauté internationale.

b) Elle soutient en priorité les efforts des pays en développement, régions et groupes de populations les plus défavorisés. Elle encourage notamment le développement rural; I'amélioration alimentaire - en particulier les cultures vivrières destinées à la consommation locale; la promotion de I'artisanat et de la petite industrie locale; la création d'emplois; la recherche et le maintien d'un équilibre écologique et démographique." 
Ce texte, qui sert de base à la formulation de la politique, met clairement l'accent sur un objectif : améliorer le sort des populations défavorisées (qui ne se trouvent pas uniquement dans les pays les plus pauvres). II prend ainsi en compte les disparités régionales et sociales qui n'apparaissent pas à la lecture des indicateurs fondés sur des moyennes nationales et qui sont hélas - les plus fréquemment utilisés.

L'objectif essentiel est donc de contribuer à une réduction des inégalités internes dans les pays du Tiers Monde, qui doit aller de pair avec la réduction des inégalités internationales. La croissance économique est considérée comme une condition nécessaire mais non suffisante à la réalisation de cet objectif : "I'expérience montre que le fort taux de croissance économique qu'ont connu certains pays n'a pas empêché la détérioration de la situation de la majorité de la population. Pire encore, une croissance économique mal orientée, qui ne viserait pas à satisfaire en priorité les besoins essentiels des populations, peut, à terme, faire obstacle à un développement véritablement centré sur les besoins de la population" (14). S'il n'y a pas de garantie que le développement profite aux plus défavorisés, "il est important que notre coopération technique et notre aide financière soient directement orientées vers les régions ou les groupes de populations qui restent en marge de l'effort de développement entrepris par leur propre gouvernement" (15).

La poursuite de cet objectif passe en premier lieu par la satisfaction des besoins essentiels de l'homme qui incluent les droits les plus élémentaires de la personne humaine. Les projets et programmes entrepris dans les différents secteurs prioritaires définis à l'article 5 de la loi doivent donc tendre à la satisfaction des besoins essentiels et, par là, à la réduction des inégalités (16).

Le maintien et la recherche d'un équilibre écologique sont des objectifs aussi directement liés à la pauvreté, aux disparités économiques et sociales. "Aujourd'hui, l'équilibre écologique est mesuré dans de nombreuses parties du monde non seulement par l'usage inadéquat des techniques agricoles et industrielles modernes, mais aussi par l'exploitation souvent abusive, quoique traditionnelle, des ressources naturelles par les groupes de populations les plus déshérités. L'application irrationnelle de certaines techniques modernes et le combat désespéré pour l'existence que mènent des groupes de populations socialement et géographiquement en marge de l'évolution se révèlent aussi dangereuses I'une que I'autre pour l'environnement naturel de I'homme" (17).

Les objectifs poursuivis prennent une nouvelle dimension avec l'introduction du concept de "self-reliance" : "On ne saurait concevoir un nouvel ordre économique international ni un régime propre à assurer la satisfaction des besoins essentiels de l'homme ou l'équilibre entre l'homme et l'environnement naturel sans comprendre dans ces notions l'idée d'un renforcement de la confiance en soi des peuples du Tiers Monde, et la perspective de plus larges possibilités d'autodétermination... Lorsqu'il s'agit d'offrir, même aux populations les plus démunies, une possibilité d'engager leurs forces aussi 
bien dans le domaine de l'économie que dans celui de la culture, la confiance qu'ont en soi les personnes intéressées et la liberté d'action qui leur est laissée prend un rôle prédominant" (18). Dans la logique de ce qui précède, la coopération suisse au développement appuiera les initiatives “ $d$ 'où qu'elles viennent et souhaite la mise sur pied de structures décentralisées, propres à encourager et à appuyer les initiatives qui peu à peu se prennent dans un pays" (19). Sur un plan plus global, cette attitude se traduit par une volonté d'encourager la coopération Sud-Sud comprise comme une voie vers une autodétermination collective.

Cette volonté de coopérer à la promotion d'un développement auto-centré et bénéficiant aux couches les plus défavorisées de la population devait amener le gouvernement à préciser, en 1980, certaines des conditions politiques de la coopération au développement : "La violation flagrante des droits de I'homme peut remettre en question la poursuite d'un programme de coopération au développement" (20). Cependant, ce n'est que dans les situations extrêmes que cette remise en question sera suivie d'effets, car le Conseil fédéral constate que la violation des droits de l'homme est souvent une circonstance qui aggrave les conditions de vie de populations déjà frappées par la misère. Dans ces conditions... "Ies projets d'assistance technique et d'aide financière qui profitent directement aux populations les plus défavorisées peuvent devenir un moyen, sinon de faire respecter tous les droits de la personne humaine, du moins d'assurer la survie de gens qui ne sont pas responsables mais victimes du comportement de leurs autorités" (21). En résumé, la doctrine officielle sur ce point est "qu'il faut continuer à prêter aide à la population sous certaines formes, lorsque cette aide contribue à atténuer les effets du non respect des droits de l'homme et non à renforcer cette politique" (22).

\section{Les objectifs quantitatifs dans le domaine de l'APD}

La faiblesse quantitative de I'APD de la Suisse est un trait persistant des relations de ce pays avec le Tiers Monde. La Suisse n'a jamais souscrit à I'objectif défini en 1970 par les Nations Unies d'atteindre un volume d'APD équivalant à $0,7 \%$ de son PNB. Tout au long de la décennie 1971-1980, la Suisse s'est assigné des objectifs plus modestes : atteindre le niveau moyen d'APD par rapport au PNB des pays industrialisés membres du CAD. II s'agissait d'abord d'y arriver vers la fin des années 1970; ce but n'ayant pas été atteint, l'échéance fut reportée au milieu de la présente décennie.

En 1977, lors de la conférence sur la coopération économique internationale (dite aussi Dialogue Nord-Sud), la Suisse fut le seul pays participant avec les Etats-Unis à refuser l'objectif du 0,7\%. Elle fut, de plus, seule à ne pas pouvoir prendre l'engagement d'accroître substantiellement son aide publique au développement.

Le plan financier de la Confédération pour les années 1981/1983 prévoyait que I'APD passerait de $0,24 \%$ en 1980 à $0,27 \%$ en 1981 et à $0,31 \%$ pour 1982 
et 1983. En fait, des coupures budgétaires affectant l'APD (18\% de réduction au lieu de $10 \%$ pour d'autres postes) firent qu'une fois de plus l'objectif fixé par la Confédération n'a pas pu être atteint. L'APD ne représentera vraisemblablement que 0,25\% du PNB en 1982 (23).

\section{Principes guidant la coopération suisse au développement}

La coopération suisse au développement comprend, selon la loi y relative, plusieurs formes : coopération technique, aide financière, mesures de politique économique et commerciale. Dans ce paragraphe, nous traiterons avant tout des mesures qui occasionnent des flux d'aide publique au développement, laissant de côté les mesures politiques et commerciales telles qu'accords sur les produits de base, préférences douanières pour les articles manufacturés en provenance des pays du Tiers Monde, etc.

\section{La répartition des flux entre l'aide bilatérale et multilatérale}

Les organisations multilatérales de coopération au développement (en particulier le Programme élargi d'assistance technique des Nations Unies qui deviendra le PNUD) jouèrent un rôle très important dans la gestion de l'APD de la Suisse jusqu'à la création, en 1961, d'un appareil étatique chargé spécifiquement de cette tâche. Jusqu'à cette date, les organisations internationales reçurent jusqu'à $90 \%$ des flux, à vrai dire modestes, engagés.

Par la suite, la part de l'aide multilatérale diminuera pour osciller dans les années 1970 entre 30 et $40 \%$ de l'APD.

La clé de répartition entre les flux bilatéraux et multilatéraux est fonction, d'une part, de la nécessité pour la Suisse de collaborer avec les organisations internationales, et d'autre part, des résistances politiques internes qui se manifestent à l'encontre de ces organisations.

Les arguments gouvernementaux en faveur de la coopération avec des organisations internationales peuvent être classés en deux catégories : ceux se rattachant aux vertus particulières de la coopération multilatérale au développement, et ceux liés à l'intérêt propre de la Suisse à poursuivre cette forme de coopération. Parmi les premiers, on relève (24) :

- "I'avantage pour la coopération multilatérale d'être moins liée à des conditions politiques que la coopération directe avec une puissance disposant d'importants moyens financiers;

- le rôle de coordination entre organismes d'aide que peuvent jouer les organisations internationales;

- le fait que la coopération multilatérale permet un accroissement du volume de l'aide sans exiger du personnel supplémentaire dans les agences bilatérales" (25). 
Mais la Suisse trouve un intérêt à maintenir cette forme de collaboration, car :

- elle peut ainsi faire valoir ses points de vue au sein des organisations internationales;

- les "entreprises suisses ont la possibilité de répondre aux appels d'offres lancés par ces organisations dans le cadre de leurs projets" (26);

- la Suisse ne peut rester à l'écart d'organisations au sein desquelles se façonne de plus en plus son avenir.

Cette batterie d'arguments en faveur de la coopération multilatérale est notamment destinée à contre-balancer les réticences provenant de divers courants d'opinions publiques à l'égard de la collaboration avec les institutions financières internationales (Banque mondiale et banques régionales de développement). Ces réticences, illustrées par le rejet lors d'une votation populaire d'un crédit de deux cents millions de francs à I'IDA, sont de nature très hétérogène et ont trait aussi bien au principe même de la coopération internationale qui empièterait sur la souveraineté nationale, qu'au faible contrôle que la Suisse pourrait exercer sur l'emploi qui est fait de ses ressources. Une autre critique réside dans le fait que la nature même des activités de banques internationales ou régionales de développement ne favorise pas le développement des groupes de populations les plus défavorisés, telle que dictée par la loi suisse sur la coopération au développement.

Au-delà du débat qui se poursuit en Suisse sur les avantages et les inconvénients de la coopération multilatérale au développement, il est certain que le rejet, en 1976, du crédit destiné à I'IDA a limité les possibilités du gouvernement d'accroître la part de cette coopération dans son programme d'APD.

\section{Répartition géographique et sectorielle de la coopération bilatérale}

Sur le plan de la répartition géographique de I'APD, il existe une tension entre la volonté des autorités suisses de voir le principe de l'universalité de ses relations s'appliquer aussi à la coopération au développement et la faiblesse des moyens financiers et humains qui ne permettent pas raisonnablement de mettre en œuvre des programmes de coopération avec tous les pays du Tiers Monde. Les contributions à des organisations internationales ont donc aussi pour objet de "toucher" indirectement des pays qui ne reçoivent pas d'aide bilatérale de la part de la Suisse.

Le principal critère guidant le choix des pays est celui du niveau de développement, la loi indiquant qu'il s'agit de soutenir en priorité les pays, régions et populations les plus défavorisés. L'accent est ainsi mis sur les pays les moins développés et sur ceux étant classés par la Banque mondiale parmi les pays à faible revenu (moins de cinq cents dollars par habitant en 1979).

Pour les crédits mixtes, le choix des pays bénéficiaires obéit à des critères en partie différents : il doit s'agir de pays trop endettés pour financer leur développement uniquement par des crédits commerciaux mais qui ont néan- 
moins un niveau de développement qui ne justifie plus des contributions d'APD exclusivement sous forme de dons. La limite supérieure du revenu moyen est fixée à mille dollars en 1978. Il faut en outre "qu'il existe un besoin réel de biens suisses dans le pays intéressé", ces crédits mixtes étant liés à I'acquisition de tels biens (27).

La répartition géographique prévue pour les années 1981-1983 est d'environ $45 \%$ pour l'Afrique, 35\% pour I'Asie et $20 \%$ pour "les pays les plus défavorisés d'Amérique latine" (28).

Dans chacun des continents, "par souci d'efficacité", la coopération suisse concentre l'essentiel de ses projets de coopération technique et d'aide financière dans un nombre limité de pays qui sont actuellement :

en Afrique : pays du Sahel (Haute-Volta, Mali, Niger, Tchad), Madagascar, Rwanda, Tanzanie, Cameroun, Kenya et Mozambique;

en Amérique latine : Pérou et Honduras;

en Asie: Bangladesh, Inde, Indonésie, Népal et Pakistan.

Dans cette liste, on trouve nombre de pays qui y figurent depuis le début des activités de la coopération suisse au développement et qui furent choisis de façon assez empirique. Une certaine prédilection pour les petits pays, montagneux, enclavés (à l'image de la Suisse?) semble avoir influencé le choix des premiers pays partenaires de la coopération suisse. Aujourd'hui, le critère de développement joue un rôle déterminant dans le choix des pays de "concentration" ainsi que les expériences faites dans la coopération bilatérale. Récemment, le coup d'Etat de juillet 1980 en Bolivie a remis en cause son statut de pays de "concentration". La Bolivie a été remplacée par le Honduras. Mais d'une manière générale, la liste est relativement stable.

Du point de vue de la répartition sectorielle, la priorité va aux domaines mentionnés dans la loi :

- développement rural

- amélioration de la situation alimentaire

- promotion de l'artisanat

- création d'emplois

- maintien de l'équilibre écologique et dêmographique.

Les projets étant souvent multisectoriels et poursuivant simultanément des objectifs liés à plusieurs de ces domaines, le Conseil fédéral ne fixe pas de règles de répartition des ressources entre secteurs ou domaines. II apparaît cependant assez clairement, de l'analyse de listes de projets de la DDA, que le développement rural représente de loin le domaine le plus important et qu'à l'intérieur de ce domaine, le secteur de l'agriculture et de l'élevage occupe une place prépondérante.

L'analyse qui sous-tend ces choix est qu'il convient de lutter contre la pauvreté en premier lieu dans le domaine rural :

- la majorité de la population pauvre des pays les plus pauvres vivant dans ce milieu; 
- le développement rural permettant de freiner l'exode vers la grande ville et d'affaiblir ainsi l'accroissement de la pauvreté urbaine.

\section{Les principes de base de la coopération bilatérale}

\section{a) Les rôles des fournisseurs et des destinataires de I'APD}

Les textes légaux et de politique gouvernementale donnent une conception assez claire de la coopération au développement. II s'agit certes de "contribuer à donner à ces pays la possibilité d'assurer leur développement par leurs propres forces". Mais encore faut-il que ces efforts soient entrepris en vue d'améliorer les conditions de vie des populations des pays en développement, et que la coopération s'adresse en priorité aux pays, régions et groupes de population les plus pauvres (voir les articles 3 et 5 de la loi).

C'est dire que le rôle du destinataire dans la détermination du programme d'aide sera d'autant plus fort que sa politique de développement poursuivra des objectifs proches de ceux définis dans la loi. Dans le cas contraire, il sera important de veiller à ce que I'APD soit "directement orientée vers les régions ou des couches de population qui restent en marge de l'effort de développement entrepris. par ces gouvernements" (29).

Dans la même optique, on souligne dans les textes officiels que les activités gouvernementales ne sont pas les seules qui doivent être soutenues par la coopération internationale au développement : "quelles que soient les structures économiques, politiques et sociales des pays en développement, les organisations bilatérales et multilatérales d'aide au développement sont toutes conscientes de la nécessité de ne pas seulement soutenir les activités gouvernementales, mais d'appuyer d'une façon ou d'une autre, toutes les initiatives, d'où qu'elles viennent. Ces organisations attendent des autorités qu'elles leur facilitent ce type d'intervention" (31). Si I'approbation des autorités nationales est toujours requise pour entreprendre des projets de coopération avec d'autres partenaires, il n'en reste pas moins que ces projets ne figurent pas nécessairement dans les priorités gouvernementales. Dans ces cas-là, le fournisseur d'aide joue un rôle déterminant dans la formulation et la mise en place du projet.

Pour le reste, la collaboration permanente avec les autorités nationales de planification est considérée comme essentielle. Dans la préparation et la gestion des projets, on tend à associer de plus en plus des ressortissants des deux pays, voire de confier d'emblée la responsabilité principale de la direction d'un projet à des représentants du pays destinataire.

\section{b) La planification à long terme}

Le gouvernement suisse est conscient de la nécessité de planifier à long terme la coopération au développement et qu'elle ne doit pas être "guidée par des considérations à court terme, ni trop influencée par les fluctuations de la conjoncture politique" (32). De fait, nous l'avons vu, la coopération avec 
les pays de "concentration" tend en général à être stable sur une longue période et la coopération suisse accepte de contribuer à long terme (dix ans et plus) au financement de projets lorsque cela s'avère utile.

Par contre, des engagements formels de soutien à des projets ou des programmes sont rarement pris pour des périodes excédant trois ans. En effet, les crédits octroyés par le Parlement fixent un plafond aux montants qui peuvent être engagés au cours d'une période minimale (en général trois ans). Ainsi des engagements financiers pris pour de plus longues périodes conduiraient à mobiliser des ressources importantes sur certains projets et programmes au détriment d'autres engagements.

Récemment, la Suisse a entrepris I'élaboration de programmes par pays qui déterminent les secteurs prioritaires de coopération à moyen terme. Deux programmes de ce type ont été établis, l'un pour I'Inde (1980-1983) et l'autre pour le Mali (1981-1983).

\section{c) La collaboration avec les ONG}

La Suisse est un pays dans lequel les ONG jouent un rôle très actif, tant dans la mobilisation de ressources pour la coopération au développement et la réalisation de projets, que dans le débat public et l'information sur les pays du Tiers Monde et les relations Nord-Sud. Ces ONG sont liées à des Eglises ou à des organismes laïques. Les plus importantes d'entre elles collaborent au sein d'un organe de coordination.

Les fonds propres recueillis par ces ONG équivalent, environ, au quart des ressources d'APD (1979-1980); cette proportion est de loin la plus élevée de tous les pays du CAD. Les projets des ONG peuvent être en partie subventionnés par la DDA, pour autant qu'ils répondent aux critères de la politique officielle de coopération au développement. Ces subventions représentent en général $50 \%$ environ du coût du projet. En 1979, elles représentèrent $10 \%$ environ de I'APD. Depuis trois ans, la DDA accorde à certaines ONG des crédits sur la base de leur programme annuel de coopération technique. En outre, les ONG les plus importantes gèrent des projets pour le compte de la DDA, ce qui permet notamment de décharger l'administration publique. En 1982, sept ONG ont, à l'initiative de la DDA, créé un nouvel organisme, "Intercoopération", essentiellement chargé de gérer des projets bilatéraux, financés entièrement par la Confédération.

Les domaines de coopération touchés par les ONG sont très divers; certaines d'entre elles sont spécialisées dans des actions sectorielles comme la santé ou la formation professionnelle. Beaucoup d'entre elles agissent dans le domaine du développement rural. En général on constate que leurs priorités sont les mêmes que celles des pouvoirs publics. Au cours de ces dernières années, plusieurs ONG ont orienté une part croissante de leurs ressources vers le soutien d'activités d'ONG de pays du Tiers Monde, en particulier vers des organisations qui cherchent à promouvoir un type de développement autocentré. 
d) Les exigences formulées à l'égard du pays destinataire de I'APD

En principe, selon la politique officielle, tout pays du Tiers Monde peut être destinataire de I'APD suisse, le seul facteur limitatif étant le niveau de PNB par habitant. II n'existe donc aucune exigence particulière quant au système économique, au régime politique des pays destinataires et de ce fait, les activités bilatérales se sont déroulées et se déroulent dans des pays représentant des types de développement économique et politique très différents.

Qu'en est-il si le gouvernement d'un pays destinataire porte atteinte à des intérêts suisses ou à des principes auxquels le gouvernement helvétique est attaché? En 1973, en présentant son projet de loi au Parlement, le Conseil fédéral répond de la manière suivante : "... il convient de partir de l'idée que les mesures que nous prenons au titre de la coopération internationale au développement se placent dans le cadre de nos intérêts au sens le plus large. Cela ne signifie toutefois pas que la coopération au développement puisse sans autre servir de moyen de pression lorsque ces intérêts et principes sont lésés. Dans de nombreux cas, on toucherait notamment en dernier ressort des populations en I'occurrence hors de cause, sans pouvoir atteindre I'effet voulu sur le gouvernement ou d'autres responsables de l'atteinte qui nous serait portée. Une atteinte qui pourrait être prise en considération serait celle qui dénature les résultats de la coopération avec la Suisse. Egalement une atteinte portée aux intérêts économiques suisses, par exemple des mesures de nationalisation sans indemnisation adéquate, peuvent être prises en considération dans ce contexte, quoiqu'il apparaisse souvent contre-indiqué de lier directement la coopération au développement avec la protection des intérêts économiques suisses. En bref, l'attitude d'un pays, qui par exemple serait contraire au droit des gens, peut difficilement rester sans influence sur nos prestations de coopération et d'aide. Dans une telle éventualité, la Suisse cherchera probablement à conduire à chef, dans la mesure du possible, les activités entreprises, mais elle fera preuve de réserve à l'égard de nouvelles activités proposées" (32).

Cette formulation souple et prudente reflète bien la pratique : à quelques reprises au cours des dernières années, différentes actions du gouvernement de pays destinataires ont conduit la Suisse à freiner plus ou moins sensiblement son programme de coopération au développement dans tel ou tel pays. Ainsi, l'explosion d'un engin atomique indien en 1974 conduisit la Suisse à freiner pendant quelques mois la mise en œuvre de nouveaux projets dans ce pays. En Bolivie, la violation systématique des droits de l'homme par le gouvernement issu du coup d'Etat militaire de juillet 1980 provoquait une réduction très sensible de l'APD suisse à ce pays.

$\mathrm{Si}$, en principe, la Suisse ne formule aucune exigence quant à la politique générale intérieure ou extérieure du gouvernement destinataire, elle ne manque pas, lors de la négociation d'accords de projets de coopération technique ou d'aide financière, de poser certaines conditions ayant trait aux objectifs du projet, ou à ses modalités de réalisation ou à son suivi. Il s'agit là de 
demandes qui sont habituellement formulées par les fournisseurs d'APD :

- intégration du projet dans les plans gouvernementaux, c'est-à-dire reconnaissance de son caractère prioritaire; assurance que les mesures éventuelles en amont ou en aval du projet sont prévues et seront réalisées en temps opportun;

- participation des partenaires au financement du projet, en particulier le coût du personnel local, des frais de fonctionnement, éventuellement d'une partie des frais d'investissement. Au cours des dernières années, I'attitude des autorités suisses sur cette question est devenue relativement souple : un co-financement est presque toujours exigé, mais on peut plus aisément négocier la nature et le volume des prestations du gouvernement destinataire :

a) assurance de prise en charge complète du projet par les organisations nationales après le retrait de la coopération internationale;

b) enfin, il sera évidemment demandé que les objectifs du projet, en particulier dans le choix des bénéficiaires, soient conformes aux objectifs généraux de la coopération suisse au développement.

Pour ce qui est des crédits mixtes, la Confédération exige que le pays destinataire dispose "d'un bon appareil administratif pour prendre des décisions concernant I'utilisation du crédit et pour régler les questions de procédure" (33).

\section{Les formes de la coopération au développement}

La coopération bilatérale revêt plusieurs formes qui obéissent chacune à des critères et à des modalités d'attribution différents.

La coopération technique ne comprend que des prestations sous forme de dons ou assimilables à des dons.

L'aide financière est attribuée soit sous forme de dons ou de prêts à des conditions de faveur.

Ces deux formes de coopération au développement s'adressent en premier lieu aux pays les plus pauvres et veulent “en priorité élever le niveau de vie des populations les plus défavorisées" (34). La coopération technique et I'aide financière, qui représentent environ le $85 \%$ de I'APD de la Suisse, sont, dans leurs composantes bilatérales, essentiellement réalisées sous forme de projets.

Les achats de biens et services financés par la Suisse dans le cadre de ces projets ne sont, en principe, pas liés à l'achat de biens ou de services suisses. Dans les projets d'aide financière, les acquisitions se font par voie d'appels d'offres internationaux, ce qui n'est pas le cas dans les projets de coopération technique où les montants destinés à des achats d'équipements sont beaucoup plus modestes. Dans ce cas, le choix des fournisseurs dépendra beaucoup des services d'entretiens offerts sur place, et de la connaissance de l'équipement que possède le personnel expatrié et national du projet. 
Les mesures de politique économique et commerciale revêtent diverses formes :

- des crédits mixtes combinant des apports du secteur public (APD) et des crédits bancaires accordés aux conditions du marché;

- des aides aux balances des paiements de pays du Tiers Monde;

- des participations à des mesures internationales de soutien aux produits de base, ou promotion commerciale bilatérale de certains produits;

- des mesures tendant à encourager l'industrialisation et

- à promouvoir les exportations de produits manufacturés des pays du Tiers Monde.

La description et l'analyse de ces mesures font l'objet d'un article d'Antoine Brawand dans cet Annuaire.

\section{L'APD de la Suisse : Que disent les chiffres?}

\section{Le volume global et les conditions de l'APD}

Une analyse du volume de l'APD de la Suisse montre un début très modeste en $1960(0,04 \%$ du PNB) et une croissance lente mais assez régulière jusqu'en 1969 (0,16\% du PNB) suivie de fluctuations dans la décennie 1971-1980, le niveau final ne parvenant pas à dépasser le cap des $0,25 \%$ (voir le tableau 1 ). Comme nous I'avons indiqué ci-dessus, le volume de I'APD atteindra au mieux 0,30\% du PNB vers le milieu de cette décennie. Comment expliquer la stagnation à ce faible pourcentage de l'APD suisse? On peut exclure les facteurs explicatifs de type économique et financier puisque la Suisse est sans conteste le pays membre du CAD qui a connu le moins de difficultés économiques depuis une dizaine d'années. Cette stagnation est sans doute due, en premier lieu, à une faible volonté politique de la part du gouvernement et du Parlement qui admettent que la coopération au développement est mal comprise et mal acceptée (35) bien qu'en principe, les partis gouvernementaux soient favorables à un volume accru de I'APD (36).

On peut aussi mentionner que les ressources des administrations publiques en Suisse sont nettement plus faibles que dans d'autres pays, tels les pays scandinaves (34\% du PNB en 1976 par rapport à $50 \%$ pour la Norvège et $57,4 \%$ pour la Suède). L'OCDE note à cet égard : “Il semble que les pays qui, sur le plan interne, ont choisi de renforcer le plus les administrations publiques soient généralement aussi favorables à une participation active du secteur public dans le domaine de l'aide et aient les moyens de l'assumer"'(37). 


\begin{tabular}{|c|c|c|c|c|c|c|c|c|c|}
\hline & 1960 & 1965 & 1970 & 1975 & 1976 & 1977 & 1978 & 1979 & 1980 \\
\hline APD (millions de \$) & 3,5 & 11,8 & 30,2 & 103,6 & 112,2 & 119,0 & 173,4 & 206,7 & 246,3 \\
\hline APD (\% PNB) & 0,04 & 0,09 & 0,15 & 0,19 & 0,19 & 0,19 & 0,20 & 0,21 & 0,24 \\
\hline $\begin{array}{l}\text { Organismes secteur } \\
\text { privé (millions } \\
\text { de \$) }\end{array}$ & $2,3^{*}$ & 3,7 & 11,2 & 32,1 & 34,2 & 34,4 & 48,6 & 51,3 & 63,2 \\
\hline$\%$ PNB & $0,02^{*}$ & 0,03 & 0,05 & 0,06 & 0,06 & 0,06 & 0,06 & 0,05 & 0,06 \\
\hline
\end{tabular}

*Chiffres pour 1961

Source : Statistiques du CAD et divers messages du Conseil fédéral

Les conditions auxquelles I'APD suisse est accordée sont en général plutôt favorables par rapport à la moyenne des pays membres du CAD. Ainsi, la part des dons dans l'ensemble de I'APD s'élevait en 1980 à 92\% (moyenne CAD 75,6\%). L'élément de libéralité du total des engagements était, la même année, de $96,9 \%$ (moyenne du CAD 90,1\%). La répartition de l'APD entre dons et prêts a évolué depuis cinq ans en faveur des dons; cela est dû au fait que depuis 1976, une part importante de l'aide financière - celle destinée aux pays les plus pauvres - est accordée à fonds perdus. La répartition actuelle (1980) est de $87,5 \%$ de dons et de $12,5 \%$ de prêts; en 1976 , les chiffres étaient $70,7 \%$ et $29,3 \%$. Aujourd'hui, les pays à bas revenus ne reçoivent plus que des dons.

Les chiffres des dernières années font apparaître des progrès dans le déliement de I'APD de la Suisse. La part de l'APD non liée a passé de $64,7 \%$ pour la période $1976-1978$ à $69,7 \%$ pour 1980 , les chiffres correspondants pour les membres du CAD sont de $49,3 \%$ et $52,8 \%$ (pour 1979) (38).

\section{La répartition des flux entre l'aide bilatérale et multilatérale}

Au cours des quinze dernières années, la répartition de I'APD entre flux bilatéraux et multilatéraux a évolué en faveur de ces derniers, ainsi que le montre le tableau II ci-après. 
Tableau II Répartition des flux d'APD entre l'aide bilatérale et multilatérale

\begin{tabular}{|c|c|c|c|c|c|c|c|c|}
\hline & $\begin{array}{l}1965-67 \\
\text { moyenne }\end{array}$ & 1970 & 1975 & 1976 & 1977 & 1978 & 1979 & 1980 \\
\hline \multicolumn{9}{|c|}{ En millions de dollars } \\
\hline APD totale & 12,6 & 30,2 & 103,6 & 112,3 & 119,0 & 173,4 & 206,7 & 246,3 \\
\hline APD bilatérale & 8,2 & 18,4 & 70,6 & 67,0 & 69,2 & 100,8 & 109,1 & 138,0 \\
\hline APD multilatérale & 4,4 & 11,8 & 33,0 & 45,3 & 49,8 & 72,5 & 97,6 & 108,3 \\
\hline \multicolumn{9}{|l|}{ En pourcentage } \\
\hline APD totale & 100,0 & 100,0 & 100,0 & 100,0 & 100,0 & 100,0 & 100,0 & 100,0 \\
\hline APD bilatérale & 65,1 & 61,0 & 68,1 & 59,7 & 58,2 & 58,2 & 57,8 & 56,1 \\
\hline APD multilatérale & 34,9 & 39,0 & 31,9 & 40,3 & 41,8 & 41,8 & 42,2 & 43,9 \\
\hline
\end{tabular}

Source : Coopération pour le développement, CAD/OCDE.

Dans son message de 1980, le Conseil fédéral annonçait cependant la volonté de ramener la part multilatérale de I'APD à $27 \%$. Cette volonté s'est immédiatement traduite au niveau de la présentation des statistiques du CAD par le transfert, dès 1980, sous la rubrique "APD bilatérale", de l'aide associée et de co-financements, c'est-à-dire des contributions de la Suisse à des organisations internationales pour la réalisation de projets spécifiquement désignés par le fournisseur d'aide. En 1980, ces contributions se sont élevées à 32,2 millions de dollars. Elles figuraient jusqu'à cette date sous la rubrique des "contributions aux organismes multilatéraux" (39).

La direction des flux multilatéraux est avant tout dictée par des facteurs institutionnels : la non-appartenance de la Suisse à la Communauté économique européenne et au groupe de la Banque mondiale. Avant 1976, la Suisse avait participé, à deux reprises en 1967 et 1971, à la reconstitution des ressources de I'IDA, en lui attribuant des prêts de cinquante-deux et cent trente millions de francs. En 1976, le refus par le peuple suisse de ratifier l'accord avec I'IDA concernant un prêt de deux cents millions de francs, mit un terme à ces contributions générales. Cependant, la Suisse participe au cofinancement de projets de I'IDA (usine d'engrais au Bangladesh, projet de développement rural en Haute-Volta...) pour montrer aux pays en développement "que nous sommes conscients de l'importance de l'aide que leur apporte cette organisation et, à nos partenaires industrialisés, que nous reconnaissons l'effort qu'ils font en participant régulièrement à la reconstitution des ressources de cette filiale de la Banque mondiale" (40). Cette politique à l'égard de I'IDA reflète une tendance générale de la politique de la Suisse qui opère une substitution entre ses contributions générales aux programmes des organismes multilatéraux de coopération au développement, et des soutiens à des projets spécifiques de ces organismes. Ces dernières contribu- 
tions apparaissant donc désormais dans les statistiques suisses sous la rubrique de I'aide bilatérale (41).

Les principaux canaux de I'APD multilatérale de la Suisse sont le PNUD et les trois banques régionales de développement dont la Suisse est membre. Les tableaux III et IV ci-après donnent la répartition entre les organisations internationales des contributions générales et des co-financements pour la réalisation de projets spécifiques. Pour des indications plus détaillées, voir tableaux de la partie statistique de cet annuaire.

Tableau III Contributions aux organisations multilatérales

(versements nets en millions de dollars)

\begin{tabular}{lrrr}
\hline & 1978 & 1979 & 1980 \\
1. Institutions des Nations Unies & 32,9 & 41,4 & 45,9 \\
2. Banques régionales de développement & 15,0 & 21,0 & 25,6 \\
3. Autres organismes multilatéraux & 8,5 & 8,7 & 4,6 \\
\cline { 2 - 4 } CONTRIBUTIONS & & & \\
MULTILATERALES TOTALES & 56,5 & 71,0 & 76,1 \\
\hline
\end{tabular}

Source : Mémorandum de la Suisse au CAD, 1980, p. 13.

Tableau IV Aide associée et co-financement en 1980

(versements nets en millions de dollars)

\begin{tabular}{lccc}
\hline & $\begin{array}{l}\text { Aide } \\
\text { associée }\end{array}$ & $\begin{array}{l}\text { Co-finan- } \\
\text { cement }\end{array}$ & TOTAL \\
1. Institutions des Nations Unies & 10,4 & 3,8 & 14,2 \\
$\begin{array}{l}\text { 2. Institutions financières } \\
\begin{array}{l}\text { 3. Instituts de recherche et } \\
\text { autres organismes internationaux }\end{array}\end{array}$ & 0,6 & 12,0 & 12,6 \\
\cline { 2 - 4 } TOTAL & 4,8 & 0,6 & 5,4 \\
\hline
\end{tabular}

Source : Mémorandum de la Suisse au CAD, 1980, p. 15. 


\section{La répartition géographique de l'APD bilatérale}

Le tableau $\mathrm{V}$ ci-dessous donne la répartition de I'APD bilatérale par continents, en incluant, pour toutes les années, l'aide associée, ce qui correspond à la pratique actuelle de la Suisse acceptée par le CAD.

Tableau V Répartition par continents de I'APD bilatérale et de l'aide associée (en pourcentage)

\begin{tabular}{lcrrrrrrr}
\hline & $\begin{array}{l}1961-67 \\
\text { cumulé }\end{array}$ & 1970 & 1975 & 1976 & 1977 & 1978 & 1979 & 1980 \\
Afrique & 46,2 & 39,3 & 23,4 & 26,2 & 34,8 & 41,8 & 36,8 & 39,0 \\
Amériques & 9,7 & 21,5 & 7,1 & 8,6 & 13,7 & 11,3 & 14,7 & 13,9 \\
Asie & 31,7 & 26,4 & 62,8 & 52,2 & 35,6 & 38,2 & 38,6 & 37,5 \\
Europe & 6,5 & 3,0 & 1,3 & 2,6 & 4,9 & 1,8 & 0,7 & 0,3 \\
$\begin{array}{l}\text { Non classée } \\
\text { géographique }\end{array}$ & 5,9 & 9,8 & 5,7 & 10,4 & 11,0 & 6,9 & 9,2 & 9,3 \\
TOTAL & 100 & 100 & 100 & 100 & 100 & 100 & 100 & 100 \\
\hline
\end{tabular}

Source : DDA, Berne.

La politique gouvernementale prévoit une répartition géographique des flux de I'APD qui tienne compte du niveau de développement - priorité aux pays "les plus défavorisés" - et de la nécessité, par souci d'efficacité, de concentrer I'APD sur un nombre relativement faible de pays. Qu'en est-il selon les statistiques?

La part de I'APD bilatérale et de l'aide associée attribuée aux pays les moins avancés - selon la liste des Nations Unies - a évolué comme suit :

$\begin{array}{lccccccc}\text { Total 1961-1967 } & 1970 & 1975 & 1976 & 1977 & 1978 & 1979 & 1980 \\ 14,3 \% & 14,3 \% & 21,6 \% & 20,3 \% & 32,5 \% & 42,9 \% & 30,7 \% & 36,9 \%\end{array}$

Source : DDA.

La part de L'APD qui est attribuée aux pays dits "de concentration" (il y en a dix-huit) s'établit comme suit au cours des trois dernières années, en pourcentage de I'APD destinée au continent auquel ils appartiennent : 
En pourcentage

$\begin{array}{llll}\text { Afrique : dix pays } & 68,6 & 60,6 & 62,9 \\ \text { Amérique latine : trois pays } & 42,5 & 36,9 & 45,6 \\ \text { Asie : cinq pays } & 83,7 & 80,0 & 70,0\end{array}$

Source : Mémorandum de la Suisse au CAD, 1980.

\section{La répartition sectorielle de l'APD bilatérale}

Les statistiques à disposition donnent les versements d'APD bilatérale par secteur économique pour la coopération technique seulement. Dans chaque secteur, sont regroupés divers domaines d'activité : production, services, formation et recherche.

Tableau VI Répartition sectorielle de la coopération technique bilatérale (en pourcentage)

\begin{tabular}{|c|c|c|c|c|c|c|c|}
\hline & $\begin{array}{l}\text { 1961-71 } \\
\text { cumulé }\end{array}$ & 1975 & 1976 & 1977 & 1978 & 1979 & 1980 \\
\hline Sylviculture & 1,0 & 6,2 & 5,9 & 4,3 & 5,6 & 5,1 & 5,9 \\
\hline Agriculture et élevage & 25,2 & 33,1 & 29,9 & 30,7 & 24,5 & 20,9 & 19,7 \\
\hline Santé & 5,3 & 1,9 & 1,9 & 2,9 & 3,9 & 2,5 & 5,4 \\
\hline $\begin{array}{l}\text { Hygiène sanitaire et } \\
\text { services sociaux }\end{array}$ & 2,9 & 5,1 & 5,9 & 4,0 & 5,2 & 11,4 & 4,7 \\
\hline Artisanat et industrie & 20,0 & 10,1 & 9,3 & 14,4 & 12,8 & 15,7 & 10,9 \\
\hline Tourisme & 3,8 & 5,4 & 5,6 & 4,0 & 3,3 & 3,7 & 1,8 \\
\hline $\begin{array}{l}\text { Education et formation } \\
\text { professionnelle }\end{array}$ & 16,6 & 12,5 & 11,8 & 13,5 & 12,1 & 13,0 & 15,2 \\
\hline Communications & 2,9 & 5,8 & 3,7 & 3,7 & 3,7 & 2,4 & 3,9 \\
\hline $\begin{array}{l}\text { Commerce, banques, } \\
\text { administration }\end{array}$ & 9,3 & 1,2 & 2,8 & 1,4 & 3,5 & 2,1 & 2,5 \\
\hline Autres secteurs & 9,1 & 6,6 & 10,6 & 6,6 & 14,9 & 9,8 & 12,5 \\
\hline Non classé & 3,9 & 12,1 & 12,6 & 14,5 & 10,5 & 13,4 & 17,5 \\
\hline TOTAL & 100,0 & 100,0 & 100,0 & 100,0 & 100,0 & 100,0 & 100,0 \\
\hline En millions de dollars & 45,3 & 25,7 & 32,1 & 34,8 & 53,8 & 72,1 & 79,1 \\
\hline
\end{tabular}

Source : DDA. 
En regroupant la coopération technique et l'aide financière, on obtient la répartition suivante pour les versements cumulés de 1961 à 1981 :

$\begin{array}{lr}\text { Agriculture et élevage } & 36,3 \% \\ \text { Sylviculture } & 5,8 \% \\ \text { Education } & 16,9 \% \\ \text { Industrie et artisanat } & 20,0 \% \\ \text { Santé et hygiène } & 14,8 \% \\ \text { Tourisme } & 6,2 \%\end{array}$

\section{Tendances actuelles de l'aide suisse au développement}

En Suisse, comme dans la plupart des pays industrialisés, la dernière décennie a incontestablement favorisé une prise de conscience accrue des milieux politiques et économiques de l'importance des relations Nord-Sud, et de la complexité des phénomènes de développement. Pour l'économie suisse, les relations commerciales et financières avec les pays du Tiers Monde jouent un rôle fondamental et I'on a très largement admis que l'aide au développement et les transferts de ressources qu'elle entraîne sont devenus une composante majeure et permanente des relations extérieures de la Suisse.

Dans ce contexte, deux problèmes principaux sont, et demeureront, au centre de la politique et de la pratique de la Suisse en matière de coopération au développement : il s'agit du niveau de l'aide publique au développement et des rapports entre la politique de coopération au développement et la politique économique extérieure tout court.

Le premier point est certainement celui qui embarrasse le plus la Suisse dans les discussions et négociations internationales consacrées aux relations NordSud. II ne faut cependant pas s'attendre à un relèvement très marqué de cet APD au cours des prochaines années. D'un côté, certaines organisations nongouvernementales "Tiers-mondistes", favorables à une APD plus importante, subordonnent cette croissance à la nécessité d'améliorer la qualité de l'aide, c'est-à-dire sa capacité à contribuer à l'amélioration durable du sort des populations les plus défavorisées. C'est dire que les milieux les plus favorables à une $A P D$ désintéressée ne font pas de l'accroissement de I'APD leur principal cheval de bataille. D'un autre côté, les partis politiques gouvernementaux qui veulent limiter l'ensemble des dépenses publiques ne sont pas favorables à une augmentation notable de I'APD, augmentation qui serait nécessaire pour que la Suisse fournisse dans ce domaine des prestations plus à la mesure de sa prospérité

La question des rapports entre politique économique extérieure et politique de coopération au développement a fait l'objet de nombreux débats et le gouvernement cherche prudemment - nous l'avons vu - dans son discours et dans sa pratique, à ménager les partisans des deux thèses que l'on peut 
schématiser ainsi :

- celle qui voudrait que l'ensemble des relations économiques extérieures de la Suisse avec les pays du Tiers Monde soient considérées à la lumière des objectifs de la loi sur la coopération au développement, et que les instruments publics de politique extérieure soient adaptés à la poursuite de ces objectifs;

- celle qui voudrait que la coopération au développement, en particulier de I'APD, soit mise de façon plus directe au service des entreprises suisses.

La première position, défendue avant tout par les ONG et des partis politiques de gauche, critique la pratique de l'aide liée, en particulier les crédits mixtes, comme une forme de promotion des exportations qui ne profite guère aux groupes de population les plus défavorisés. Elle est aussi réservée à l'égard des Banques internationales de développement, dont les projets seraient peu aptes à améliorer le sort de ces mêmes groupes de populations. Elle voudrait aussi que la garantie publique des risques liés á l'exportation de biens et de services ne soit pas accordée aux exportations qui ne seraient pas conformes aux objectifs de la coopération au développement.

La seconde position est celle de représentants des entreprises exportatrices. Devant les difficultés croissantes rencontrées du fait de la conjoncture économique mondiale actuelle, les partisans de cette position voudraient que I'Etat leur apporte un soutien accru en confiant plus de tâches, dans la réalisation de projets financés par I'APD ou en finançant des études de factibilité de projets susceptibles d'être ensuite réalisés par des entreprises suisses (42).

L'analyse de la pratique gouvernementale de ces dernières années fait apparaître que malgré les difficultés des entreprises exportatrices, le liement de I'APD a diminué au cours des cinq dernières années. Une mesure directe a cependant été prise pour favoriser les entreprises d'exportation, en augmen tant sensiblement le montant des crédits mixtes prévus pour les prochaines années. Le montant prévu de cent quarante millions de dollars sur trois ans reste cependant relativement modeste. La garantie publique pour couvrir les risques de l'investissement des entreprises suisses dans les pays du Tiers Monde est relativement peu utilisée par les investisseurs, qui considèrent les primes à payer trop élevées. Le financement, par la Suisse, d'un bureau de I'ONUDI à Zurich, est une autre mesure destinée à promouvoir les investissements dans le Tiers Monde, ainsi que la création d'un fonds de financement des études de pré-investissement de projets industriels de petites et moyennes entreprises suisses dans le Tiers Monde.

La tendance actuelle semble donc être un maintien de l'équilibre des années passées entre les divers objectifs de la coopération au développement. II est vrai que la situation actuelle est particulièrement favorable à la Suisse, puisque les retombées des activités de coopération au développement pour I'économie suisse sont extrêmement élevées. Ceci est notamment dû aux liens de la Suisse avec les grandes banques de développement : alors que 
I'aide financière de la Suisse à ces banques était d'environ quarante et un millions de dollars en 1980, ces banques et la Banque mondiale achetaient, en Suisse, cette même année, pour près de deux cent vingt millions de dollars de biens et de services (43).

Ce rapport coûts/bénéfices, très favorable à la Suisse, devrait inciter les autorités à chercher à améliorer encore ces prochaines années la qualité de son APD - déliement, conditions financières, participations accrues aux dépenses locales et récurrentes des projets - de façon à ce que le faible volume serve de façon optimale les intérêts des bénéficiaires dans les pays du Tiers Monde.

\section{NOTES}

1. Banque Mondiale, Rapport sur le développement dans le monde 1981, Washington, août 1981, $167 \mathrm{p}$.

2. OCDE, Coopération pour le développement, Examen 1981, Paris 1981, 263 p.

3. Une exception à cette règle : le 8 décembre 1981, lors d'un vote au Parlement sur une réduction de $18 \%$ des crédits d'APD, le parti socialiste fut le seul parti gouvernemental à s'opposer à cette mesure.

4. Nous n'analysons pas dans le cadre de cet article les contributions au titre de l'aide humanitaire. Elles sont incluses dans I'APD dont elles représentaient le $11,5 \%$ en 1980.

5. Rapport concernant les répercussions des récents événements économiques mondiaux sur la contribution de la Suisse à la coopération internationale au développement du 22 janvier 1975, p. 28.

6. Message concernant la continuation de la coopération technique et de l'aide financière en faveur des pays en développement du 9 juillet 1980, p. 22.

7. Rapport concernant les répercussions des récents événements économiques mondiaux sur la contribution de la Suisse à la coopération internationale au développement du 22 janvier 1975, p. 29.

8. Message à l'appui d'un projet de loi sur la coopération au développement et l'aide financière internationale, p. 23.

9. Voir l'Annuaire Suisse-Tiers Monde 1981, IUED, Genève, 1981, p. 98.

10. Message du 9 juillet 1980, p. 12.

11. Message du 9 juillet 1980 , pp. 22 et 23.

12. Message à l'appui d'un projet de loi sur la coopération au développement du 19 mars 1973, p. 24.

13. Message du 9 juillet 1980, p. 23.

14. Message du 9 juillet 1980, p. 24.

15. Message du 9 juillet 1980, pp. 24 et 25.

16. Il me paraît intéressant de citer dans ce contexte le contenu que donne le gouvernement à la lettre e de l'alinéa 2 de l'article 5 de la loi sur l'encouragement de la recherche et du maintien d'un équilibre démographique : "Il ne suffit pas d'interdire, d'ordonner et de conseiller pour freiner une croissance démographique lourde de conséquences. Les hommes défavorisés du Tiers Monde aussi doivent pouvoir compter sur leur santé et leur force de travail, ainsi que sur la survie de tous leurs enfants. Ils 
doivent être assurés que leurs descendants disposeront de possibilités de formation et d'emplois et que, par là, ces enfants pourront plus tard prendre soin de leurs parents. Ces conditions étant remplies, la sécurité et la confiance en soi pourront se développer sans le soutien d'une nombreuse progéniture. C'est pourquoi les améliorations apportées à la vie quotidienne par nombre de projets de coopération au développement contribuent grandement à rétablir I'harmonie entre la croissance démographique et les possibilités économiques des pays en développement" (Message du 27 septembre 1976, p. 30).

17. Message du 23 novembre 1977, p. 16.

18. Message du 23 novembre 1977, p. 18.

19. Message du 9 juillet 1980, p. 26.

20. Message du 9 juillet 1980, p. 27.

21. Message du 9 juillet 1980, p. 27. Le 15 août 1980, quelques semaines après la publication de ce texte, le Conseil fédéral appliquait cette politique en renonçant à la mise en œuvre et à la poursuite de certains projets de coopération en Bolivie, comme conséquence du coup d'Etat militaire de juillet 1980.

22. Notons cependant qu'en 1977 et 1981, la Suisse ne fut pas le seul pays à ne pas accroître substantiellement le volume de son APD par rapport au PNB.

23. Estimation fournie par le Message du 14 décembre 1981 concernant la continuation des mesures de politique économique et commerciale au titre de la coopération au développement, p. 37.

24. Les citations de ce paragraphe sont tirées du Message du 23 novembre 1977 concernant la continuation de la coopération technique et de l'aide financiére en faveur des pays en développement, pp. 41 et 42.

25. Cet argument est particulièrement pertinent en Suisse où l'effectif des fonctionnaires de l'administration fédérale a été bloqué à son niveau de 1974.

26. On verra au chapitre 5 que les entreprises suisses bénéficient effectivement largement de cette possibilité.

27. Message du 14 décembre 1981, pp. 29 et 30.

28. Voir Message du 9 juillet 1980, pp. 47 et 48.

29. Message du 9 juillet 1980, p. 25 . C'est nous qui soulignons.

30. Message du 9 juillet 1980, p. 26.

31. Message du 27 septembre 1976, p. 20.

32. Message du 19 mars 1973, pp. 35 et 36.

33. Message du 14 décembre 1981, p. 29

34. Message du 9 juillet 1980, p. 88.

35. En 1970, lors d'une enquête sur l'attitude des Suisses face à l'aide au développement, $34 \%$ des personnes interrogées étaient pour un accroissement de l'APD, $48 \%$ pour son maintien à son niveau d'alors $(0,15 \%$ du $\mathrm{PNB})$ et $15 \%$ pour une réduction de l'aide.

36. Voir à cet égard J.J. de Dardel, La coopération au développement. Certitudes et interrogations, IUED, Genève, 1981, $405 \mathrm{p}$.

37. OCDE, Coopération pour le développement, Examen 1978, p. 151.

38. Toutes les données de ce paragraphe sont tirées des statistiques du CAD.

39. Dans le tableau II, nous avons conservé I'ancienne présentation statistique pour garder I'homogénéité de la série chronologique. Dans la nouvelle présentation, la part multilatérale de I'APD tombe à 30,9\% pour 1980 .

40. Message du 9 juillet 1980, p. 48.

41. Il faut également relever qu'à la suite de nouvelles dispositions du CAD, les contributions aux organisations internationales non-gouvernementales ne figurent plus 
- dès 1980 - dans la rubrique APD multilatérale. En 1980, pour la Suisse, il s'agit essentiellement d'une contribution de dix-huit millions au Comité international de la Croix-Rouge (CICR).

42. Voir J. Forster, La nouvelle contestation de la coopération suisse au développement in Annuaire Suisse-Tiers Monde 1981, pp. 213 à 222.

43. Voir la réponse du Conseil fédéral du 7 octobre 1981 au postulat du conseiller aux Etats Generali. 